\title{
Ein weit verbreiteter Hiatus in spätglazialen Seesedimenten: Mögliche Ursache für Fehlinterpretation von Pollendiagrammen und Hinweis auf klimatisch verursachte Seespiegelbewegungen
}

\author{
Hartmut Usinger *)
}

\begin{abstract}
Critical review, lacustrine sediment, erosion, Upper Pleistocene (Alleroed-Dryas), misinterpretation, pollen diagram, climate effect, changes of sealevel, peat bog, inventory, section.

Northwestern German Plain (Kubitzbergmoor, Scharnhagener Moor, Rabensbergmoor), Schleswig-Holstein. TK 1119, 1526. Denmark (Bornholm, Vallensgard Mose)
\end{abstract}

Kurzfassung: Spätglaziale Seeablagerungen von verschiedenen Lokalitäten (hauptsächlich Schleswig-Holstein; Dänemark) lassen einen übergangslosen Wechsel von Alleröd- zu Dryas3-Sedimenten erkennen. Das Fehlen von Übergangssedimenten weist auf einen Hiatus. Erosionserscheinungen an der Oberfläche der Alleröd-Sedimente bestätigen die Vermutung. Pollenanalytische Untersuchungen zeigen, daß der Hiatus bedeutende Teile von Alleröd und Dryas-3 umfassen kann, und daß dies nicht in plötzlichen Veränderungen von Pollenhäufigkeiten im Diagramm zum Ausdruck kommen muß. Im Zuge der Hiatus-Bildung kann aufgearbeitetes Alleröd-Material in Dryas-3-Sediment gelangen und dessen Pollenflora entstellen. Die Ursache der Hiatus-Entstehung dürfte in einer vorübergehenden Seespiegelsenkung und/oder anderen klimatisch bedingten Phänomenen zu suchen sein. Aus Beobachtungen anderer Autoren ist zu schließen, daß der Hiatus weit über das Gebiet bisheriger Nachweise hinaus erwartet werden muß.

[A Widespread Hiatus in Late Weichselian Lake Sediments: A Possible Cause for Misinterpretation of Pollen Diagrams and an Indication of Climatically Controlled Water Level Changes]

A bstract : Late-weichselian lake deposits from several sites (mainly in Schleswig-Holstein, West Germany. Denmark) show an abrupt change from Alleröd to Younger Dryas sediments. The absence of transitional sediments points to a hiatus. Marks of erosion on the surface of the Alleröd layers, as observed at some sites, confirm this assumption. Pollenanalytical investigations show that the hiatus may include essential parts of Alleröd and Younger Dryas layers, and this may not be reflected by sudden changes of pollen frequencies in the diagrams. When the hiatus was formed, reworked Alleröd material was deposited together with Younger Dryas sediments, and can distort their pollen flora. The hiatus seems to be caused by a lowering of the water level and/or other phenomena controlled by climate. Observations published by other authors indicate that the hiatus can be expected to exist far beyond the area where it has been recorded until now.

\section{Einleitung}

Lagerungsstörungen in Seesedimenten sind seit langem bekannt und wiederholt an Einzelbeispielen und zusammenfassend beschrieben worden (z. B. LUNDQvisT 1924; Schütrumpf 1936; Müller 1969; Ammann-Moser 1975; Averdieck \& Prange 1975; Nichols 1967). Ihre Folgen für die Interpretierbarkeit stratigraphischer Befunde sind offensichtlich: Nicht rechtzeitig erkannt, können sie Ergebnisse langwieriger Untersuchungen wertlos machen; nicht erkannt, führen sie fast zwangsläufig zu Fehlinterpretationen. So muß jede Lagerungsstörung interessieren, in der mehr als ein lokales Phänomen vermutet werden kann.

*) Anschrift des Verfassers: Dr. H. U s in g e r, Botanisches Institut der Universität, Olshausenstraße 40-60, D-2300 Kiel. 
Bei pollenanalytisch-stratigraphischen Untersuchungen an spätglazialen Seeablagerungen in Schleswig-Holstein (Usinger 1975) ist zwischen den Sedimenten des Alleröd und denen der Jüngeren Tundrenzeit (im Folgenden: Dryas-3) ein Hiatus erkannt worden. Seine Nachweisbarkeit an mehreren gut aufgeschlossenen Spätglazial-Vorkommen des Landes ließ auf ein zumindest regional verbreitetes Phänomen schließen.

Dieser Schluß hat sich inzwischen als richtig erwiesen: es liegen sowohl neue Nachweise wie auch Beobachtungen anderer Autoren vor, nach denen mit gutem Grund eine weitere Verbreitung des Hiatus angenommen werden kann. Darüber hinaus hat sich nachweisen lassen, daß der Hiatus weit größere Teile von Alleröd und Dryas-3 umfassen kann, als es aufgrund bisheriger Befunde scheinen mochte. Dies hat speziell für die Deutung des allerödzeitlichen Abschnitts betroffener Diagramme erhebliche Konsequenzen.

Die vorliegende Arbeit soll in erster Linie auf die (mögliche) Existenz eines Hiatus im genannten Niveau hinweisen. Mit der Beschreibung seiner Merkmale an verschiedenen Lokalitäten sollen Anhaltspunkte gegeben werden, ihn auch anderenorts zu erkennen. Der Frage nach den Ursachen soll nachgegangen werden. Nicht beabsichtigt ist, bei der Zusammenstellung von Hinweisen auf die Existenz des Hiatus, die sich aus Beobachtungen anderer Autoren ergeben, Vollständigkeit zu erzielen. -

Mein herzlicher Dank für Hilfe bei Grabungsarbeiten gilt meinen Freunden und Kollegen Priv. Doz. Dr. F. Albers, Prof. Dr. Kl. Müller, cand. rer. nat. K. Weinert und Dipl.Biol. A. Wolf. Frau Dr. H. Carmesin danke ich für vielfältige Hilfe bei der Anfertigung des Manuskripts.

\section{Beobachtungen und Ergebnisse}

Im Folgenden werden zunächst von verschiedenen Spätglazial-Vorkommen lithostratigraphische Befunde beschrieben, die zur Feststellung des Hiatus geführt haben. Diesen Befunden werden korrespondierende pollenanalytische Ergebnisse gegenübergestellt. So wird beurteilbar, inwieweit sich die lithostratigraphisch erkannten Hiatus im Pollendiagramm widerspiegeln. Mehrere weitere Lokalitäten, von denen zwar lithostratigraphische Hiatus-Nachweise, nicht aber pollenanalytische Resultate vorliegen, wurden nicht berücksichtigt.

Für die Erkennung und Deutung des Hiatus sind die Ergebnisse wiederholter Untersuchungen im Kubitzbergmoor/Kiel von besonderer Bedeutung. Die älteren Befunde (UsINGER 1975: 94-108) werden anschließend zusammengefaßt, die neueren in Abschnitt 2.6. dargestellt.

\subsection{Kubitzbergmoor}

(Jungmoränengebiet nördl. Kiel, Schleswig-Holstein)

Das Moor beherbergt eine spätglaziale Schichtfolge in „klassischer“ 3-Gliederung: zwischen tundrenzeitlichen Absätzen aus hellgrauem, tonreichen Silt liegt der durch vorwiegend dunkle Farben deutlich abgehobene Alleröd-Horizont (vgl. Abb. 4). Dieser besteht in flachen Teilen des Sedimentationsraumes aus Algengyttja, in tieferen aus einem 2 fachen Wechsel von Algen- und Kalkgyttja. Das ältere Präboreal ist durchgehend als Algengyttja entwickelt.

Die Sedimente wurden von einem ursprünglich ausgedehnteren und tieferen, abflußlosen See abgesetzt. Nach früh-postglazialer Seespiegelsenkung und Abbau hangender Torfe liegen sie aber heute in so geringer Tiefe, daß sie an vielen Stellen durch Grabung aufgeschlossen werden konnten. 

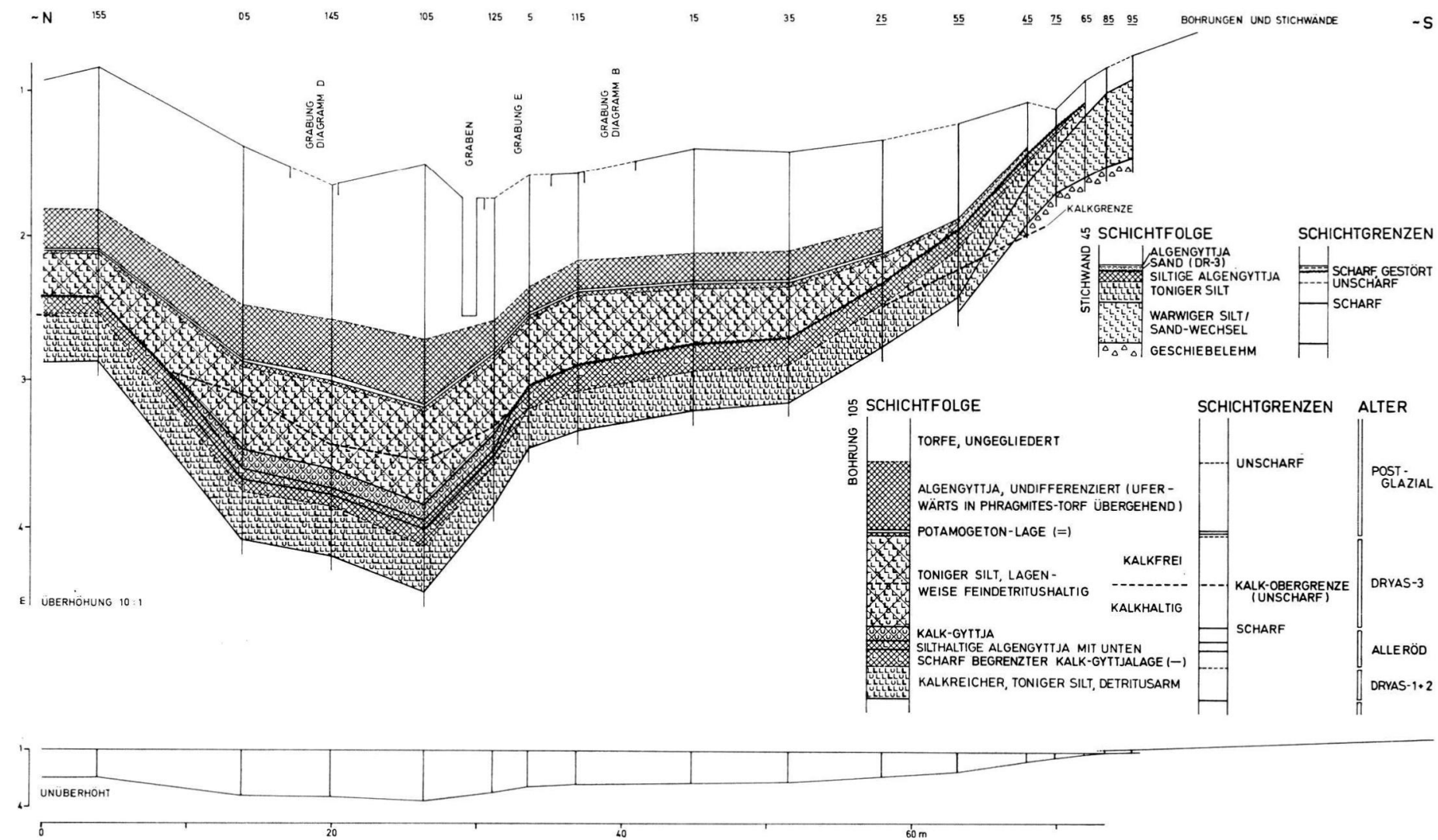

Abb. 1: Kubitzbergmoor nördl. Kiel. Stratigraphie des Spätglazials und frühen Postglazials im Südostteil des Moores (nach Usinger 1975, verändert). Sediment-Signaturen nach TroELs-SMITH (1955). 
Schon ein erster solcher Aufschluß („Grabung Diagramm B“ in Abb. 1) lieferte einen deutlichen Hinweis auf einen Hiatus: Die Grenze zwischen Alleröd-Algengyttja und Dryas-3-Silt ist auffallend scharf; im Gegensatz zu den zwar \pm schnellen, doch fließenden Sedimentveränderungen an der Untergrenze des Alleröd und Präboreal erfolgt der Wechsel hier übergangslos. Übergangssedimente, wie sie dem sicher nicht schlagartigen Wechsel von Alleröd- zu Dryas-3-zeitlichem Klima entsprächen, waren offenbar entweder abgetragen oder gar nicht erst abgesetzt. Weitere Beobachtungen - z. T. in ufernäheren Aufgrabungen (vgl. Abb. 1) - bestätigten die Existenz eines Hiatus, indem sie eindeutige Belege für Erosion allerödzeitlichen Sediments erbrachten. So wurden im Silt über der Grenzfläche Schmitzen und kleine Schollen der Algengyttja gefunden. Schichtfugen, die beim Antrocknen der Stichwände sichtbar wurden, ließen lokale Winkeldiskordanzen zwischen Schichtung und Oberfläche der Algengyttja erkennen: eindeutige Zeichen also für unterschiedlich starken Abtrag. Sand in Linsen oder dünner Lage auf der Oberfläche der Algengyttja ließ sich als deren Erosionsrückstand deuten.

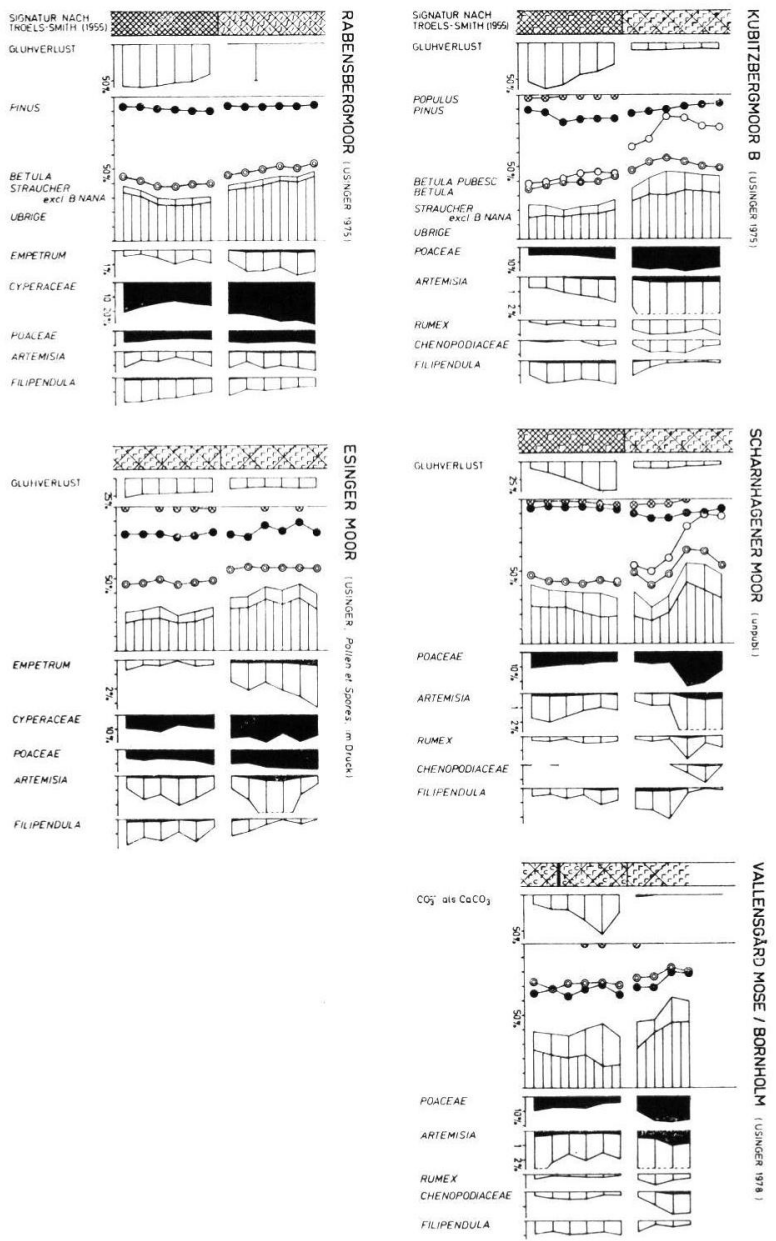

Abb. 2: Häufigkeit wichtiger Pollentaxa beiderseits des Hiatus zwischen Alleröd- und Dryas-3Sediment. (Alle Kurven unausgeglichen. Birkentrennung nach Usinger 1978b.) 
Bohrungen, durch die tiefer liegendes Spätglazial erschlossen wurde, zeigten, daß eine scharfe Grenzfläche zwischen Alleröd- und Dryas-3-Sediment bis in die tiefsten untersuchten Teile des Sedimentationsraumes erkennbar bleibt. (Entsprechend dem oben beschriebenen Alleröd-Fazieswechsel liegt Dryas-3-Silt dort auf Kalkgyttja!) Auch im Tieferen war also spät-allerödzeitliches und eventuell früh-Dryas-3-zeitliches Sediment entweder abgetragen oder nicht abgesetzt worden.

Der unvermittelte Wechsel von Alleröd- zu Dryas-3-Sediment ließ einen ebenso plötzlichen Wechsel in der Pollenflora vermuten. Wenigstens konnte erwartet werden, daß Pollen temperaturabhängiger Taxa im Bereich des Hiatus sprunghafte Häufigkeitsveränderungen zeigen. Indessen läßt das Pollendiagramm (Auszug in Abb. 2) derartige Häufigkeitsveränderungen nur bei einem Teil dieser Pollen-Typen erkennen und dies in nur undeutlicher Weise.

So war zunächst geschlossen worden, daß der Umfang des Hiatus gering, das Alleröd also fast vollständig erfaßt sei. Der Verlauf der Glühverlustkurve (Abb. 2) stützte die Annahme. Eine Kontrolluntersuchung an einem Profil aus größerer Tiefe erschien überflüssig (vgl. aber Abschnitt 2.6.).

Natürlich weist ein schlagartiger Wechsel zwischen verschiedenartigen Sedimenten nicht notwendig auf einen Hiatus. So erfolgt beispielsweise der 2malige Übergang von Algen- zu Kalkgyttja innerhalb der tieferliegenden allerödzeitlichen Absätze des Kubitzbergmoores ebenfalls zumeist ganz unvermittelt; die Grenzflächen sind vielfach gestochen scharf (vgl. Usinger 1975, Phot. 6). Es wechseln aber warmzeitliche Sedimente einander ab; Hinweise auf Erosion an den Grenzflächen wurden nicht beobachtet; an kontinuierlicher Sedimentation kann kaum ein Zweifel bestehen. Entscheidendes Indiz für den hier beschriebenen Hiatus ist also - neben Erosionsmerkmalen - der unvermittelte Wechsel von warm- zu kaltzeitlichem Sediment („warm"- und „kalt"zeitlich hier in ranglosem Sinne).

\subsection{Scharnhagener Moor}

(Jungmoränengebiet nördl. Kiel)

Hier liegen - nach früh-postglazialer Seespiegelsenkung - ähnlich gute Aufschlußverhältnisse vor wie im benachbarten Kubitzbergmoor. Sie waren besonders günstig, als vor Jahren ein Entwässerungsgraben angelegt worden war, an dessen Wänden der dunkle Algengyttja-Horizont des Alleröd über Dm verfolgt werden konnte (vgl. Overbeck 1975: 413, dort auch ein Photo des Profiles). Schon damals war die scharfe Grenze der Algengyttja gegen den hangenden Dryas-3-Silt aufgefallen und als Ausdruck eines Hiatus gedeutet worden. Spätere Untersuchungen erbrachten im Bereich der Grenzfläche dieselben aus dem Kubitzbergmoor beschriebenen Erosionsmerkmale.

Angesichts dieser Befunde zeigt ein inzwischen vorliegendes Pollendiagramm (UsingER, in Vorbereit.; Auszug in Abb. 2) ein unerwartetes Bild: Schlagartige Häufigkeitsveränderungen temperaturabhängiger Taxa finden nicht im Niveau des Hiatus statt, sondern deutlich darüber! Ein (Birken-)Großrestdiagramm zeigt dasselbe. Die lithostratigraphische Grenze zwischen Alleröd und Dryas-3 (vgl. die unvermittelte Abnahme des Glühverlustes in Abb. 2) fällt also nicht mit der biostratigraphischen zusammen. Wie in den Abschnitten 3 und 5 erläutert, kann diese Diskrepanz sehr wahrscheinlich darauf zurückgeführt werden, daß der Dryas-3-Silt unmittelbar oberhalb des Hiatus größere Anteile umgelagerten Alleröd-Materials enthält.

Diagrammvergleiche lassen erkennen, daß der analysierte Algengyttja-Horizont aus dem Scharnhagener Moor nur den ersten Teil des Alleröd umfaßt. Es fehlen ihm Spektren mit wenigstens geringfügig erhöhten Kiefernwerten, wie sie für das jüngere Alleröd 
der Region bezeichnend sind [vgl. Abschn. 4 (3)]. Hingegen weist der älteste Dryas-3Silt geringfügig erhöhte Kiefernwerte (und höhere Filipendula-Werte) auf; er spiegelt die Existenz von Schichten des jüngeren Alleröd vor. Das Pollendiagramm läßt also eher ein vollständiges Alleröd vermuten; es liefert zumindest dort keine Hinweise auf einen Hiatus, wo er nach lithostratigraphischen Befunden liegt.

\subsection{Vallensgård Mose}

(Bornholm, Dänemark. Vgl. Usinger 1978a)

In den spätglazialen Absätzen dieses Moores, die nur als Bohrkerne vorlagen, wurde die scharfe Grenzfläche zwischen Alleröd- und Dryas-3-Sediment wohl nur deshalb erkannt, weil auf sie geachtet worden ist: Sie trennt hellgraue, siltreiche, elastische Kalkgyttja von fast gleichfarbenem, kalkarmen, plastischen Silt (vgl. die $\mathrm{CaCO}_{3}-\mathrm{Kurve}_{\text {in }}$ Abb. 2).

Sichere Merkmale für Erosion allerödzeitlichen Sediments waren am vorliegenden Material nicht zu erkennen. Das Fehlen von Übergangssedimenten ist damit praktisch der einzige lithostratigraphische Hinweis auf einen Hiatus. Daß trotzdem an dessen Existenz kaum ein $Z_{w}$ eifel bestehen kann, ergibt sich aus den folgenden Indizien:

(1) Das Pollendiagramm (Auszug in Abb. 2) zeigt im Niveau der Grenzfläche für zahlreiche Taxa erhebliche Häufigkeitsveränderungen.

(2) Ein älteres Diagramm aus dem Vallensgård Mose (Iversen 1954), das die Verhältnisse tiefer liegender und mächtigerer Schichten wiedergibt, belegt für das späte Alleröd Kiefernwerte über 40\%. Dagegen endet der Anstieg der Kiefernkurve im hier betrachteten Diagramm bereits bei Werten um $30 \%$.

(3) Laacher Bimstuff, der nach verschiedenen Diagrammen aus dem mittleren Deutschland [vgl. Abschn. 4 (2)] kurz nach dem Beginn der hier mittel- bis spät-allerödzeitlichen Kiefern-Ausbreitung niederging, liegt im Profil aus dem Vallensgård Mose unmittelbar unter der Oberkante des Alleröd-Sediments.

\subsection{Rabensbergmoor}

(Altmoränengebiet südöstl. Süderlügum, Schleswig-Holstein. Vgl. Usinger 1975: 83-93)

Auch hier ist das wesentliche lithostratigraphische Indiz für einen Hiatus ein übergangsloser Wechsel von Alleröd- zu Dryas-3-Sediment, und zwar folgt auf plastische Feindetritusgyttja ein grober Braunmoos-(Scorpidium-)Schwemmtorf. Auf der Grenzfläche liegt vielfach Feinsand in Linsen oder dünner Lage.

Die Grenzfläche kann von den flachen Randbereichen der Hohlform bis in das Tiefste verfolgt werden. Das Pollendiagramm (Auszug in Abb. 2), dem ein Profil aus dem Tiefsten zugrunde liegt, belegt im Bereich der Grenzfläche für mehrere Taxa plötzliche Häufigkeitsveränderungen. So scheint es, daß die Grenzfläche auch im Tiefsten einen Hiatus markiert; selbst hier müßte also vorübergehend Abtrag erfolgt oder doch Sedimentation unterbunden gewesen sein. Daß indessen nur Sediment des ausgehenden Alleröd in geringer Mächtigkeit fehlen dürfte, geht aus dem ausgedehnten Diagrammabschnitt mit erhöhten Kiefernwerten hervor.

\subsection{Esinger Moor}

(Altmoränengebiet südöstl. Elmshorn, Schleswig-Holstein)

Die spätglazialen Sedimente entstammen einem maximal etwa $2 \times 3 \mathrm{~km}$ großen See von nur wenigen $\mathrm{m}$ Tiefe, der bereits im frühen Postglazial weitgehend verlandet bzw. einer Seespiegelsenkung zum Opfer gefallen war (vgl. Grube 1957, Hallik \& Grube 1954). 
So konnten ursprünglich relativ tiefliegende Schichten aufgegraben und einer eingehenderen Untersuchung unterzogen werden (Usinger, Pollen et Spores, im Druck).

Als weitgehend störungsfrei erwiesen sich nur die vor-allerödzeitlichen Absätze. Aufwärts treten wiederholt scharfe und z. T. gestörte Grenzflächen auf, und zwar innerhalb des Alleröd-Horizonts, an dessen Obergrenze und innerhalb der präborealen Schichten. Alle diese Grenzflächen dürften auch nach pollenanalytischem Befund Schichtlücken markieren.

Hier sei nur auf den Alleröd/Dryas-3-Hiatus näher eingegangen. Er trennt - grob betrachtet - Algen- von Siltgyttja. Beide Sedimente liegen aber nicht in reiner Form, sondern in Mischung mit Anteilen des jeweils anderen vor: Die Algengyttja ist zur Kontaktfläche hin zunehmend in plattige Bruchstücke aufgelöst, zwischen die - offenbar epigenetisch - Siltgyttja gedrungen ist. Die Siltgyttja über der Kontaktfläche führt diffus und lagenweise schon makroskopisch erkennbare, kantige Algengyttja-Stücke.

Damit unterscheiden sich die Sedimente beiderseits des Hiatus im Esinger Moor ganz erheblich von den zuvor beschriebenen Beispielen. Ursache dürften extreme Flachwasserbedingungen sein, die es ermöglichten, daß während Dryas-3 Frost mit seinen vielfältigen Folgen (vgl. Nichols 1967) bis in die Sedimente vordringen konnte (Näheres bei Usinger 1. c.). Daß das Pollendiagramm (Auszug in Abb. 2) beiderseits des Hiatus nur unbeträchtliche Häufigkeitsveränderungen zeigt, ist angesichts der beschriebenen Sedimentdurchdringung verständlich.

Der Umfang des Hiatus ist schwer abzuschätzen. Einerseits läßt das Pollendiagramm einen späteren Alleröd-Abschnitt mit deutlich erhöhten Kiefernwerten erkennen. Andererseits sind selbst die maximal erreichten Kiefernwerte mit 20\% deutlich niedriger, als im Bereich des Esinger Moores zu erwarten. So muß zumindest in Betracht gezogen werden, daß beträchtliche Teile des späten Alleröd nicht erhalten geblieben sind.

\subsection{Neue Untersuchungen im Kubitzbergmoor}

Wie oben ausgeführt, haben ältere Untersuchungen im Kubitzbergmoor zwar eindeutige lithostratigraphische Belege für einen Alleröd/Dryas-3-Hiatus erbracht, gleichzeitig hat aber ein erstes Pollendiagramm (B) den Eindruck erweckt, daß dieser Hiatus einen nur ganz unbedeutenden Abschnitt des späten Alleröd umfasse. Hinweise, daß dieser Eindruck trüge - so vor allem die geringe Tiefenlage des analysierten Profils im Vergleich zu den tiefstgelegenen Nachweisen von Erosionserscheinungen an Alleröd-Sedimenten - waren nicht weiter verfolgt worden. Dies geschah nun erst im Anschluß an folgende Befunde von anderen Spätglazial-Lokalitäten:

Mehrere Pollendiagramme aus dem südlichen Skandinavien (DEgerbøl \& Krog 1959, Berglund 1966, Andersen 1980 u.a.) weisen für das Alleröd eine deutlich 2gipfelige Birkenkurve aus; offenbar sind die allerödzeitlichen Betula pubescens-Wälder dieses Gebietes durch eine Temperatur-Depression inmitten des Alleröd vorübergehend stärker gelichtet worden; es kann eine 3-Gliederung des Alleröd ( $a, b, c)$ vorgenommen werden. In Schleswig-Holstein ist ein Birkenrückgang, der dem Alleröd-b mit hoher Wahrscheinlichkeit zugeordnet werden kann, allenfalls in nördlichen Landesteilen deutlich. Süd(ost)wärts hat die Temperatur-Erniedrigung dieser Zeit die (Moor-)Birke anscheinend nicht mehr wesentlich beeinträchtigt.

Nun wurde bei Untersuchungen in der Eichholz-Niederung bei Heiligenhafen (UsıNGER 1978b) gefunden, daß dort zwar nicht die Birke, wohl aber die Pappel (Populus tremula) inmitten des Alleröd einen deutlichen Rückgang zeigt; die Pappelkurve weist zwischen Maxima im frühen und späten Alleröd ein ausgeprägtes Minimum auf. Trotz 


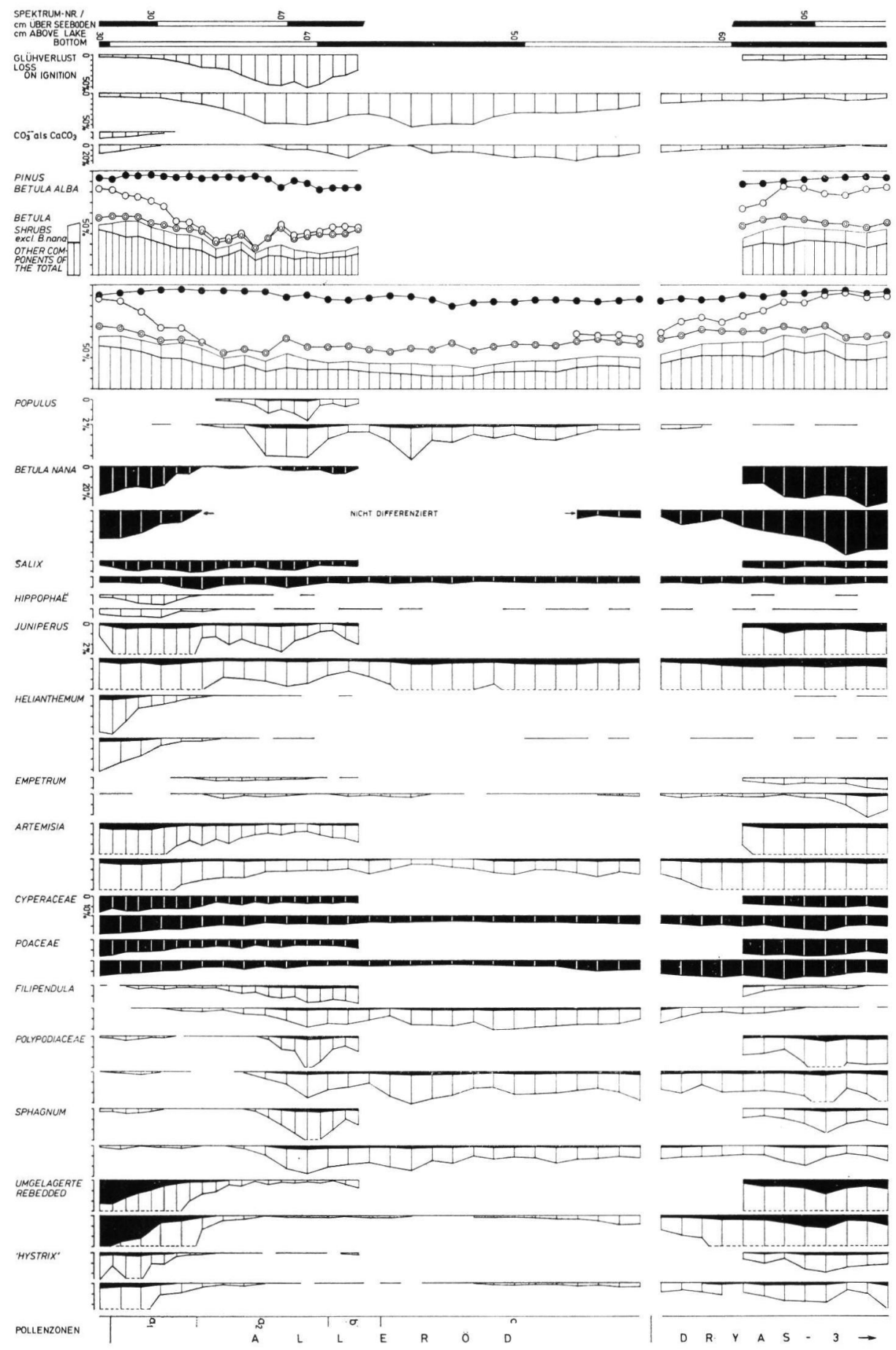

Abb. 3: Kubitzbergmoor nördl. Kiel. Konnektierung der Diagramme B (links; Usinger 1975, verändert) und $D$. 
praktisch gleichbleibender Birkenwerte kann dieses Minimum als Ausdruck einer Abkühlung gewertet werden; der Schluß steht sowohl mit dem gegenüber Betula pubescens geringfügig höheren Wärmebedürfnis der Zitter-Pappel in Einklang, er findet darüber hinaus Bestätigung in der Tatsache, daß das Pappel-Minimum mit leicht erhöhten Nichtbaumpollen-Werten und minimalen Juniperus-Werten (vgl. Usinger 1978a: 22) zusammenfällt. So konnte vermutet werden, daß sich die Temperaturdepression des Alleröd-b in Schleswig-Holstein in Rückgängen von Pappel und Wacholder nachweisen lasse.

Das Diagramm Kubitzbergmoor B (Usinger 1975. Vgl. Abb. 3) entsprach nun gar nicht dieser Vorstellung. Der mittlere Teil seines allerödzeitlichen Abschnitts - damals als Alleröd-b gewertet - ist nicht durch Populus- und Juniperus-Minima, sondern -Maxima ausgezeichnet. Die Vorstellung eines durch Minima dieser Taxa charakterisierten Alleröd-b konnte allenfalls dann aufrecht erhalten werden, wenn angenommen wurde, daß das genannte Diagramm ein nur sehr unvollständiges Alleröd umfasse.

Genau diese Annahme konnte nun durch ein weiteres, aus tiefer liegenden Schichten stammendes Diagramm ( $\mathrm{D}$ in Abb. 3, vgl. Abb. 1) bestätigt werden. Im Einzelnen:

(1) Das neue Diagramm zeigt eine deutlich 2gipfelige Populus-Kurve. Das PopulusMinimum koinzidiert mit einem Juniperus- (und einem Glühverlust-)Minimum. Damit erscheint die Alleröd-Gliederung des Diagrammes Eichholz-Niederung bestätigt und zugleich die des Diagrammes Kubitzbergmoor B widerlegt.

(2) Die Diagramme B und D lassen sich zweifelsfrei konnektieren. Damit ist sowohl die Existenz eines bedeutenden Hiatus in B erwiesen, wie auch dessen Umfang näher bestimmt: Er umfaßt neben Teilen des Alleröd-b das gesamte Alleröd-c, ferner unbekannte Abschnitte von Dryas-3.

Die Konnektierung der allerödzeitlichen Diagramm-Abschnitte erfolgte vor allem nach dem Verlauf der Kurven von Juniperus, Helianthemum, ArtemisialFilipendula, Polypodiaceae, Sphagnum. (Angenäherte) Parallelität der Kurven konnte allerdings nur erreicht werden, indem die Diagramme in verschiedenen Tiefenmaßstäben dargestellt wurden: B wurde gegenüber D um Faktor 0.67 „gestaucht“. Die größere Häufigkeit von Populus-Pollen in D beruht auf höherer Sicherheit in der Diagnose von schlecht erhaltenen Pollenkörnern.

Die Konnektierung der Dryas-3-zeitlichen Diagramm-Abschnitte erfolgte im wesentlichen nach den Ergebnissen der größenstatistischen Birkenpollen-Trennung: In Diagramm B erfolgte der Wechsel von Betula pubescens- zu B.nana-Dominanz - angezeigt durch einen Wechsel von negativschiefen Pollengrößen-Verteilungen zu positiv-schiefen - unmittelbar oberhalb des Hiatus; im Diagramm D liegt dieser Wechsel deutlich höher (Näheres hierzu bei der Veröffentlichung des vollständigen Diagrammes D).

(3) Die Konnektierung der beiden Diagramme erklärt, warum beim Diagramm B aus dem Verlauf der Glühverlustkurve auf ein fast vollständig erfaßtes Alleröd geschlossen werden konnte: Der am Ende des allerödzeitlichen Abschnitts dieses Diagrammes verzeichnete Rückgang des Glühverlustes war als end-allerödzeitlich aufgefaßt worden, liegt aber in Wahrheit am Beginn des Alleröd-b. Die Fehlinterpretation des Diagrammes B beruhte also nicht unwesentlich auf dem Zufall, daß das Alleröd der zugrundeliegenden Schichtfolge ausgerechnet bis zu diesem stratigraphisch 2deutigen Niveau abgetragen worden war. -

So eindeutig die Konnektierung der beiden Diagramme auch scheinen mag, es gab vorübergehend - Zweifel an ihrer Richtigkeit. Sie ergaben sich aus der oben erwähnten Tatsache, daß Parallelität der Häufigkeitskurven nur zu erreichen ist, indem Diagramm B beträchtlich gestaucht wird. Hieraus folgt, daß in flacheren Teilen des Sedimentationsraumes (Diagr. B) eine deutlich höhere Sedimentationsrate angenommen werden muß, als in tieferen Teilen (Diagr. D), und dies wiederum widerspricht der Regel, nach der - bei 
nichtlitoralen Sedimenten - mit zunehmender Tiefe zunehmende Mächtigkeiten zu erwarten sind. Um Klarheit zu gewinnen, wurde zwischen den zur Entnahme der Profile B und $\mathrm{D}$ vorgenommenen Aufgrabungen eine weitere durchgeführt ( $\mathrm{E}$ in $\mathrm{Abb} .1)$. An einer gut $4 \mathrm{~m}$ langen, dem Fallen der Schichten folgenden Wand (Abb.4) war nun u. a. zu erkennen:

(1) Aufwärtiges Auskeilen von jüngeren Alleröd- und älteren Dryas-3-Schichten.

(2) Abwärtige Abnahme der Mächtigkeit aller innerhalb der Alleröd-Gyttja farblich klar geschiedenen und dadurch gegeneinander abgrenzbaren Horizonte.

Beobachtung (1) belegt die nach den Diagrammen zu fordernde schnelle aufwärtige Vergrößerung des Hiatus. Beobachtung (2) bestätigt die nach der Konnektierung der Diagramme zu fordernde abwärtige Abnahme der (Alleröd-)Sedimentmächtigkeit und dies auch in betragsmäßig ausreichendem Umfang.

Der Frage nach den Ursachen der abwärtigen Abnahme der Sedimentationsmächtigkeit soll hier nicht weiter nachgegangen werden. In Betracht kommen neben primär unterschiedlichen Sedimentationsraten epigenetische Vorgänge wie stärkere Kompaktion der noch heute sehr wasserreichen Algengyttja durch höhere Auflast aber auch Streckung der Horizonte beim Einsinken über schwindendem Toteis. Im übrigen beobachtete auch KROG (1954) eine geringfügige Abnahme der Mächtigkeit des Alleröd-Horizontes mit zunehmender Tiefe.

\section{Ursachen und Zeitraum der Entstehung des Hiatus}

Beide Fragen sind anhand der damals vorliegenden Beobachtungen bereits von UsinGER (1975) erörtert worden. Hier folgt eine Darstellung unter Berücksichtigung der neuen Befunde.

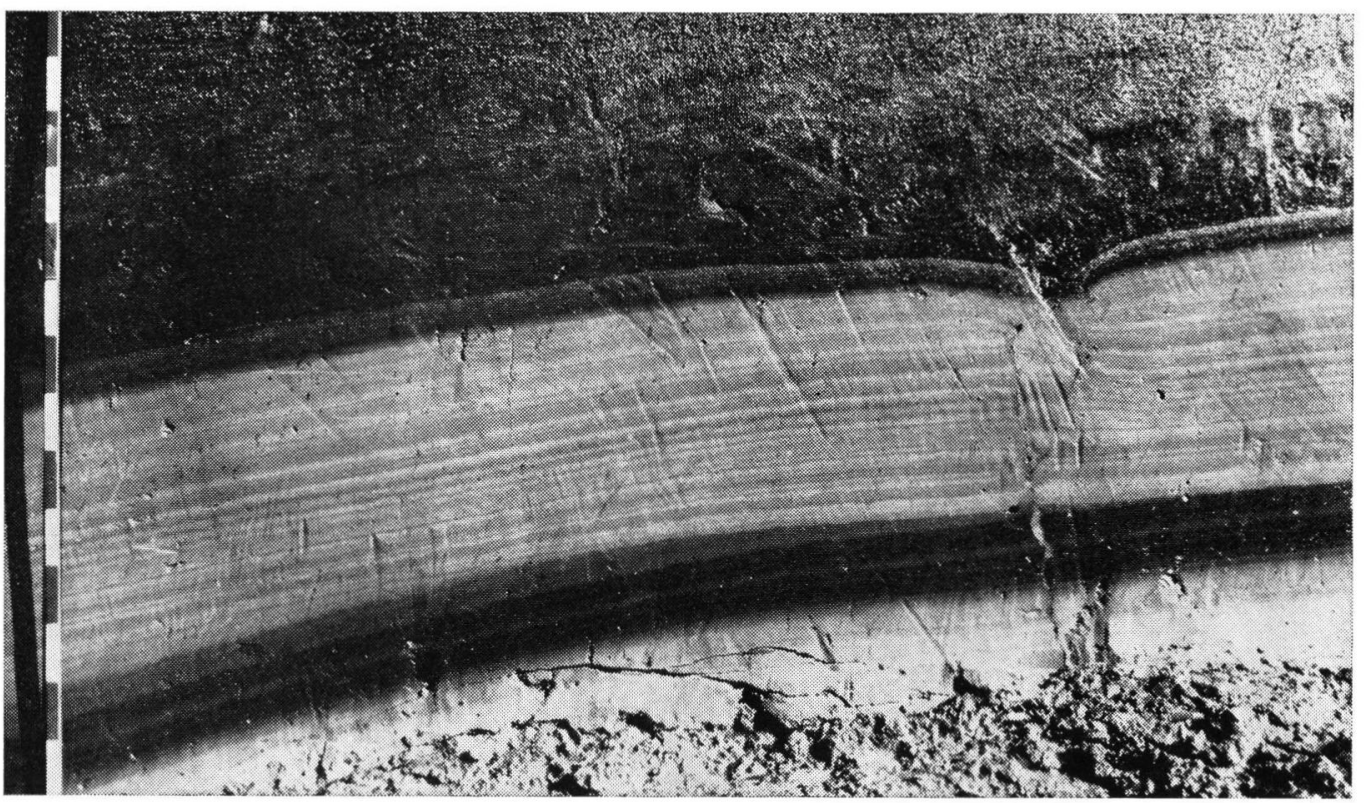

Abb. 4: Kubitzbergmoor. Spätglaziale und früh-postglaziale Schichtfolge in Grabung E (Abb. 1). Dunkle Lage: Alleröd-Gyttja. Aufwärtiges Auskeilen von spät-Alleröd- und früh-Dryas-3-zeitlichen Schichten. Maßstabsabschnitte: $10 \mathrm{~cm}$. 
Permanente Sedimentation, die zu konsolidierten Sedimenten führt, findet in Seen nur unterhalb der sog. Sediment(ations)grenze statt. Oberhalb dieser Grenze können vorübergehend unkonsolidierte Sedimente entstehen, auf Dauer überwiegt hier Erosion. Die Lage der Sedimentgrenze wird nicht nur durch Welleneinwirkung bestimmt, sondern bis weit darunter hinab durch Bodenströmungen, die aus windinduzierten Oberflächenströmungen resultieren (vgl. LUNDQvisT 1924).

Wenn konsolidierte Seesedimente lokal über die Sedimentgrenze geraten und erodiert werden, kann dies auf lokalen Verlagerungen der Bodenströmungen (in der Horizontalen) beruhen. Wenn aber flächenhaft Erosion oder doch Sedimentationsunterbrechung stattfindet, muß eine generelle Absenkung der Sedimentgrenze angenommen werden. Deren Ursache kann in einer Seespiegelsenkung, aber auch darin bestehen, daß Bodenströmungen infolge einer Veränderung der thermischen Verhältnisse des Sees oder infolge höherer Windgeschwindigkeiten tiefer und/oder dauerhafter hinabsetzen.

Die beschriebenen Beobachtungen an der Grenzfläche Alleröd-/Dryas-3-Sediment zeigen, daß der Hiatus Folge von Erosion (oder Sedimentationsunterbrechung) ist. Dichte Beobachtungsnetze in einem Teil der untersuchten Sedimentationsräume belegen flächenhafte Ausdehnung dieser Phänomene bis in größere Tiefe. An fast allen anderen Lokalitäten, wo auf sie geachtet worden ist, konnten sie trotz z. T. nur punktuellen Aufschlusses nachgewiesen werden. All dieses weist darauf hin, daß der Hiatus Resultat einer generellen Absenkung der Sedimentgrenze ist.

Die bislang informativsten Beobachtungen über Ursachen und Ausmaß dieser Absenkung der Sedimentgrenze stammen aus dem Kubitzbergmoor: Hier liegen über den höchstgelegenen, weitgehend abgetragenen Alleröd-Algengyttjen Dryas-3-Flachwassersedimente in Form von groben, z. T. kiesigen Sanden (vgl. Abb. 1). In diesem Niveau hat also Abtrag eines Stillwassersediments durch Abrasion, d. h. Wellen- (und Eis-)einwirkung in flachem Wasser stattgefunden. Abwärts folgt über weniger weit abgetragener AllerödGyttja fast unvermittelt Dryas-3-Stillwassersediment in Form von tonreichem Silt. Gröberes Korn (Mittelsand) findet sich nur als dünne Lage oder in Linsen auf der Grenzfläche zwischen den Sedimenten. Dieser Sand kann neu herangeführt, aber auch Erosionsrückstand fortgeführter Alleröd-Gyttja sein. Seine geringe Mächtigkeit läßt vermuten, daß in diesem Niveau Abrasion bereits ausklang und die Erosion des Alleröd-Sediments eventuell allein auf Bodenströmungen beruhte. In noch größerer Tiefe (in Abb. 1 nur teilweise erfaßt) liegt tonreicher Dryas-3-Silt unmittelbar auf Alleröd-Gyttja. Flachwassersedimente fehlen völlig. Sofern hier Abtrag von Alleröd-Gyttja stattgefunden hat (und nicht nur Sedimentationsunterbrechung), dürfte er allein auf Bodenströmungen zurückzuführen sein.

Abrasion von Alleröd-Stillwassersediment - wie oben beschrieben - zeigt anscheinend zweifelsfrei, daß die Absenkung der Sedimentgrenze zumindest teilweise das Resultat einer Seespiegelsenkung gewesen ist. Daß gleichzeitig mit hoher Wahrscheinlichkeit weitere Ursachen angenommen werden müssen, geht aus folgenden Überlegungen hervor:

Zwischen der höchstgelegenen, weitgehend abradierten Alleröd-Gyttja und den tiefstgelegenen Zeichen für Erosion bzw. Sedimentationsunterbrechung liegt im Kubitzbergmoor eine vertikale Spanne von mindestens $4 \mathrm{~m}$. Im Rabensbergmoor beträgt der entsprechende Wert etwa $3 \mathrm{~m}$. Sofern diese Spannen sich nicht nach der Entstehung des Hiatus vergrößert haben (was im Kubitzbergmoor infolge präborealen Tieftauens möglich, im Rabensbergmoor dagegen unwahrscheinlich erscheint), bedeutet dies Absenkung der Sedimentgrenze um mindestens 4 bzw. $3 \mathrm{~m}$. Es liegt zunächst nahe, diese Beträge auf eine Seespiegelsenkung gleichen Betrages zurückzuführen. Das aber erscheint aus folgendem Grund kaum möglich: Wird über den höchstgelegenen Alleröd-Gyttjen im Kubitz- und Rabens- 
bergmoor eine Wasserbedeckung von 1-2 $\mathrm{m}$ angenommen (wie sie zur Bildung dieser Sedimente selbst unter Berücksichtigung der geringen Größe der ehemaligen Seen mindestens erforderlich erscheint), so ergibt sich ein Seespiegelniveau, das nach dem heutigen Relief der Seerandbereiche nahezu ein Höchstniveau ist; bei nur wenig höherem Wasserstand würde Überlauf erfolgen. Da die höchstgelegenen Alleröd-Gyttjen ausweislich ihres Erhaltungszustandes im Zuge der Seespiegelsenkung sicher nicht trockengefallen sind, kann diese kaum mehr als 1-2 m betragen haben. Zur Erklärung der Tatsache, daß die Sedimentgrenze offenbar um einen deutlich höheren Betrag abgesunken war, müssen also sehr wahrscheinlich die oben genannten oder noch andere Ursachen herangezogen werden.

Es bleibt die Frage nach der Bildungszeit des Hiatus bzw. der Zeitstellung der ihn (mit)verursachenden Seespiegelsenkung. In tieferen Teilen der untersuchten Sedimentationsräume (Kubitzbergmoor, Vallensgård Mose, Rabensbergmoor) blieb im Liegenden des Hiatus selbst jüngeres Alleröd-c erhalten. Damit ergibt sich hier als frühest möglicher Zeitraum für den Beginn der Hiatus-Bildung das ausgehende Alleröd-c. Wo wenig Alleröd-Sediment abgetragen oder nicht abgesetzt wurde, dürfte auch nur wenig Dryas-3-Sediment fehlen. D. h. im Tieferen müßte die Sedimentation bereits in der frühen Dryas-3 wieder eingesetzt haben. Der Hiatus entstand hier an der Wende Alleröd/Dryas-3.

In geringerer Tiefe, wo jüngeres und schließlich auch mittleres Alleröd fehlt, muß in Betracht gezogen werden, daß die Bildung des Hiatus entsprechend früher eingesetzt haben kann. Wahrscheinlicher erscheint aber, daß das aufwärtige Auskeilen von jüngerem und mittlerem Alleröd im wesentlichen nicht auf aufwärts früher einsetzender, sondern aufwärts zunehmend intensiver Erosion beruht. Daß der Beginn der Hiatus-Bildung eine ge wis s e abwärtige Verzögerung erfahren hat, folgt aus der Tatsache, daß die verursachenden Vorgänge kaum schlagartig eingesetzt haben dürften.

Ganz entsprechend weist das Ende der Hiatus-Bildung eine a u f wärtige Verzögerung auf: während im Tiefsten der Absatz von Dryas-3-Sedimenten bereits wieder ein-
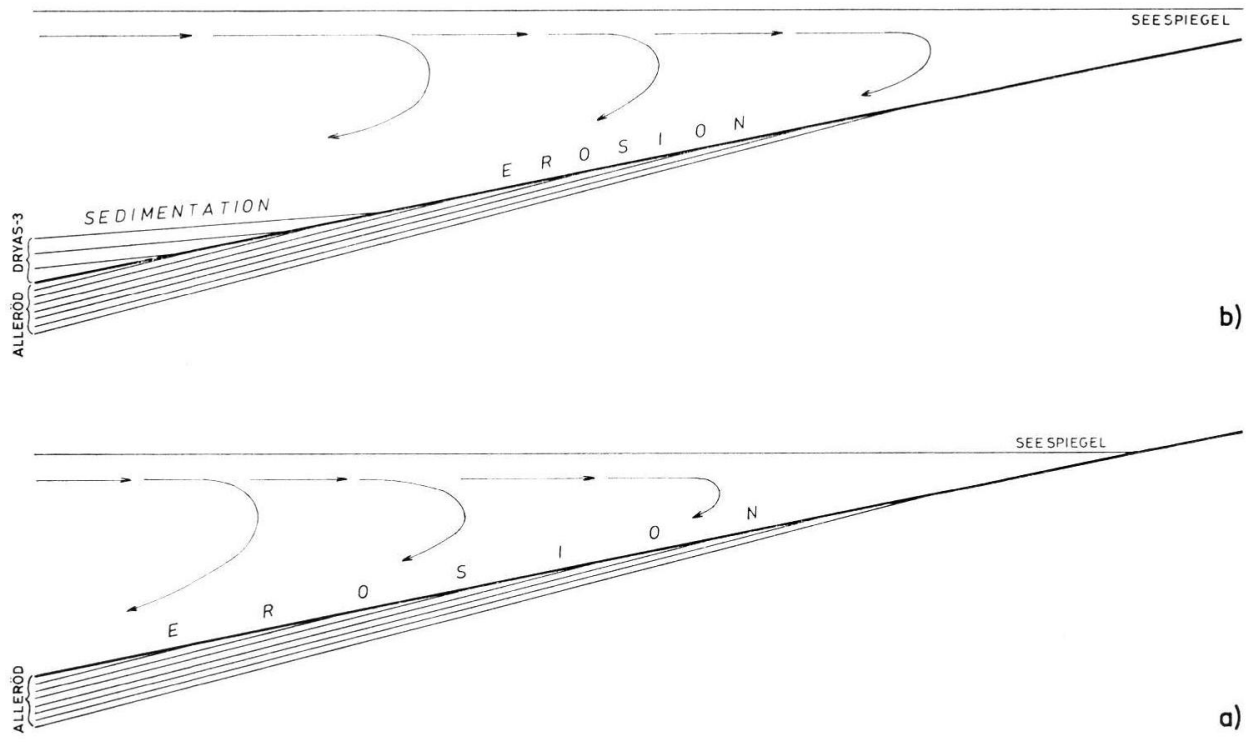

Abb. 5: Erosion und Sedimentation während bzw. nach der Hiatus-Bildung (nach Beobachtungen im Kubitzbergmoor/Kiel). Pfeile: Windstau- bzw. Bodenströme. a) Erosion von Alleröd-Sediment bis in größere Tiefe an der Wende Alleröd/Dryas-3. b) Aufwärts fortschreitende Bildung von Dryas-3-Sedimenten bei anhaltender Erosion von Alleröd-Sediment. 
gesetzt hatte, fand in geringerer Tiefe durch anhaltenden Abtrag von Alleröd-Sedimenten noch Erweiterung des Hiatus statt (vgl. Abb. 5). Nach Beobachtungen im Kubitzbergmoor scheint Erosion von höchstliegendem Alleröd sogar bis an das Ende von Dryas-3 stattgefunden zu haben und erst mit einem früh-präborealen Seespiegelanstieg beendet worden zu sein (Algengyttja des frühen Präboreal über Dryas-3-Sanden, vgl. Abb. 1, Stichwand 45). Das dürfte heißen, daß der Seespiegeltiefstand, der den Hiatus (mit)verursachte, sein Maximum bereits an der Wende Alleröd/Dryas-3 erreichte, aber abgeschwächt bis zum Präboreal andauerte. Es kann aber auch nicht ausgeschlossen werden, daß es zwischen dem maximalen Tiefstand am Ende des Alleröd und einem relativ niedrigen, die beschriebenen Flachwasserabsätze hinterlassenden Niveau am Ende von Dryas-3 Hochstände gegeben hat (vgl. Usinger 1975).

Eine Seespiegelsenkung während Dryas-3 stünde mit der Tatsache in Einklang, daß kältere Abschnitte des Pleistozän vielfach durch hohe Trockenheit ausgezeichnet waren (z. B. Frenzel 1967). Ein Widerspruch bestünde dagegen in der verbreiteten Annahme, daß die Dryas-3-zeitliche Ausbreitung von Empetrum (und Sphagnum) auf ein feuchteres Klima weise [v. D. Hammen 1953; Wijmstra 1978. Auch Cleveringa et al. (1977) vertreten diese Auffassung, obwohl sie selbst Hinweise auf eine Seespiegelsenkung während Dryas-3 gefunden haben.] Bemerkenswert ist in diesem Zusammenhang, daß Empetrum in den meisten Diagrammen größere Häufigkeit erst in der mittleren und späten Dryas-3 erreicht, d. h. also nach der vermuteten maximalen Seespiegelsenkung.

Widersprüche ergeben sich aber auch aus lithostratigraphischen Befunden: Verschiedene Autoren (SchütrumpF 1936; Steinberg 1944; Wasylikowa 1964 u. a.) fanden Dryas-3-Mudden über Alleröd-Torfen, klare Belege also für eine Seespiegelhebung während Dryas-3, sofern epigenetisches Absinken der Torfe durch Salzauslaugung, Toteistauen u. ä. ausgeschlossen werden kann. So bedarf die oben versuchte Deutung der HiatusEntstehung zweifellos der Überprüfung. Dabei wird auch der Frage nachzugehen sein, inwieweit grundsätzlich andere Ursachen als die hier angesprochenen in Betracht gezogen werden müssen. Gedacht ist vor allem an die hinsichtlich ihres (heutigen) spezifischen $\mathrm{Ge}-$ wichtes oft inverse Lage von Alleröd- und Dryas-3-Sediment, ferner an die höhere Löslichkeit von Kalk im kalten Dryas-3-Wasser, die kalkhaltige Alleröd-Sedimente selbst in konsolidiertem Zustand leicht erodierbar gemacht haben dürfte, u. dgl.

\section{Hinweise auf einen Alleröd/Dryas-3-Hiatus in Arbeiten anderer Autoren}

Die Durchsicht pollenanalytisch-stratigraphischer Arbeiten zum Spätglazial ergibt, daß von mehreren Autoren im Grenzbereich Alleröd/Dryas-3-Sediment Beobachtungen gemacht oder Ergebnisse erzielt worden sind, die mit mehr oder minder großer Wahrscheinlichkeit mit einem Hiatus in diesem Niveau erklärt werden können. Gelegentlich ist diese Möglichkeit bereits von den Autoren in Betracht gezogen worden. In kaum einem Fall kann aber von einem Nachweis gesprochen werden. [Der von Ammann-Moser (1975) sicher erkannte Hiatus greift zwar bis zum Alleröd hinab, umfaßt aber Schichten bis weit in das Postglazial, ist also nicht mit dem hier behandelten vergleichbar.] Die folgende $\mathrm{Zu}$ sammenstellung umfaßt nur solche Beispiele, bei denen sich der Verdacht auf einen Hiatus auf mehr als plötzliche Häufigkeitsveränderungen im Pollendiagramm stützt.

(1) Im Diagramm Köhlmoor (TIDELSKi 1960. Jungmoränengebiet Schleswig-Holsteins) ist durch Unterbrechung der Kurvenzüge an der Grenze zwischen Alleröd (Algengyttja) und Dryas-3 („Gyttja braun“) ein Hiatus markiert. Es fehlt allerdings jede Kommentierung. $\mathrm{Da}$ sprunghafte Veränderungen von Pollenhäufigkeiten nicht sonderlich deutlich sind, kann vermutet werden, daß TIDELSKI aus einem übergangslosen Sedimentwechsel auf einen Hiatus geschlossen hat. 
(2) Von Brande (1980) sind aus West-Berlin zwei Diagramme veröffentlicht worden, die in der Lage des eingeschalteten Laacher Bimstuffes auffällige Unterschiede zeigen: Im Diagramm Pechsee liegt der Tuff etwa $2 \mathrm{~cm}$ unter der Grenze Alleröd/Dryas-3, d. h. fast am Ende der $15 \mathrm{~cm}$ mächtigen Alleröd-Sedimente. Im Diagramm Tegeler See folgt über dem Tuff $1 \mathrm{~m}$ Alleröd-Sediment (dessen Gesamtmächtigkeit ist unbekannt, da ältere Teile fehlen).

Der erhebliche Unterschied kann z. T. auf höhere Sedimentationsraten im (am Profilentnahmeort) tieferen Tegeler See zurückgeführt werden: wie ein Vergleich der Mächtigkeiten von Dryas-3 und Präboreal in beiden Diagrammen zeigt, wurde hier 7.2 bzw. 6.7, d. h. rund $7 \mathrm{mal}$ mehr Sediment abgesetzt, als im Pechsee. Galt dieser Unterschied auch im späten Alleröd, so wäre über dem Tuff im Pechsee aber immer noch etwa $15 \mathrm{~cm}$ Sediment zu erwarten. Die tatsächlich beobachtete Mächtigkeit von $2 \mathrm{~cm}$ kann also nur durch eine im späten Alleröd drastisch erniedrigte Sedimentationsrate (vgl. Brande 1980: 33) oder auf Abtrag spät-allerödzeitlichen Sediments zurückgeführt werden.

Ein Vergleich der allerödzeitlichen Diagrammabschnitte spricht für die 2. Deutung: Das Diagramm Tegeler See zeigt im späten Alleröd ein ausgeprägtes, die Kiefernkurve deutlich überragendes Birken-Maximum; das Diagramm Pechsee läßt nichts Vergleichbares erkennen.

Ein Hinweis darauf, daß dem Diagramm Pechsee spät-allerödzeitliche Abschnitte fehlen, ergibt sich natürlich auch aus dem Vergleich mit all den anderen Diagrammen, in denen der Laacher Tuff nicht am Ende, sondern in oder wenig über der Mitte des Alleröd liegt (Steinberg 1944; H. Müller 1953; Firbas 1954; Dietz \& Grahle \& Müller 1958 u. a.). Andererseits muß auf eine Reihe von Diagrammen hingewiesen werden, nach denen der Tuff ebenfalls an das Ende des Alleröd verschoben erscheint (H. M. Müller 1961a, b, 1970 u. a.; Tuff-Nachweise südlich des Laacher Sees seien hier nicht betrachtet). Es sei dahin gestellt, ob auch in diesen Diagrammen spätes Alleröd nicht erfaßt wurde. Nur im Fall Vallensgård Mose (vgl. Abschn. 2.3) kann dies bislang als erwiesen gelten.

(3) Die für weite Teile Mitteleuropas charakteristische Ausbreitung der Kiefer im mittleren bis späten Alleröd kann aufgrund der starken Pollenproduktion und leichten Verwehbarkeit des Kiefernpollen weit über das damalige Verbreitungsgebiet des Baumes hinaus nachgewiesen werden. Nordwärts ist sie - dicht analysierte Diagramme mit hohen Grundsummen vorausgesetzt - mit Sicherheit bis in das nördliche Schleswig-Holstein (Usinger 1975; Usinger \& Wolf, in Vorb.) und von dort über die Dänischen Inseln (Degerbøl \& Krog 1959; Andersen 1980) bis in das südliche Schweden (Berglund 1966 u. a) zu verfolgen. Selbst für das mittlere Jütland (Böllingsö) zeigen 2 von 3 vorliegenden Diagrammen (IvERSEN 1942; KROG, unpubl.) deutlich erhöhte mittel- bis spät-allerödzeitliche Kiefernwerte.

So erstaunt, daß das dicht und auf hohe Grundsummen ausgezählte Diagramm Ruds Vedby/Seeland (KROG 1954) für den gesamten allerödzeitlichen Diagrammabschnitt praktisch gleichbleibende Kiefernwerte ohne jede Aufwärtstendenz ausweist. Dies könnte bedeuten, daß spät-allerödzeitliche Sedimente nicht erfaßt worden sind. Der Vergleich der Juniperus-Kurve von Ruds Vedby mit der in anderen dänischen Diagrammen scheint diesen Verdacht zu stützen, wie umgekehrt weder ${ }^{14} \mathrm{C}$-Daten noch Lithostratigraphie - die jüngsten Alleröd-Sedimente sind Driftgyttjen — Gegenargumente liefern.

(4) Bei pollenanalytisch-stratigraphischen Untersuchungen im Gebiet des ehemaligen Rosenheimer Sees/Oberbayern findet BEUG (1976: 385), daß „Durch die Klimaverhältnisse während der Jüngeren Tundrenzeit (II) ... die allerödzeitliche Vegetation offenbar nicht wesentlich beeinflußt" wurde und bestätigt hiermit Befunde aus anderen Teilen Süd- 
deutschlands. Gleichzeitig macht BEUG aber auf ein bemerkenswertes lithostratigraphisches Phänomen aufmerksam: „In auffälliger Diskrepanz zu diesen geringfügigen Veränderungen [der Vegetation] gegenüber II steht die lithostratigraphische Signifikanz der in der Zone III [ = Dryas-3] gebildeten Sedimente. Durch weißlichgraue Tongyttjen oder stärkeren Tongehalt in Kalkgyttjen hebt sich der Bereich der Zone III in den Sedimenten auffällig und mit scharfen Grenzen ab; eine einleuchtende Erklärung dafür fehlt noch.“

Nach diesen Angaben erscheint nicht ausgeschlossen, daß ein Hiatus an der Wende Alleröd/Dryas-3 auch in Seesedimenten Süddeutschlands auftritt. Unbeantwortet bleibt indessen die Frage, warum sich die klimatische Ungunst von Dryas-3 im Sediment anscheinend stärker ausgeprägt hat als in der Vegetation.

\section{Konsequenzen des Alleröd/Dryas-3-Hiatus für die (bio)stratigraphische Untersuchung des jüngeren Spätglazials}

Sie ergeben sich (1) natürlich aus der Tatsache, daß eine Alleröd- und Dryas-3-zeitliche Schichtfolge unbekannter Mächtigkeit fehlen kann und (2) daraus, daß Erosion im Zuge der Hiatus-Bildung zur Umlagerung älteren Sediments, d. h. zu dessen erneuter Ablagerung in jüngeren Schichten führt.

Der Umfang des Hiatus nimmt erwartungs- und beobachtungsgemäß mit zunehmender Tiefenlage der Schichtfolge ab. Im Tieferen eines Sedimentationsraumes - wo Proben für stratigraphische Untersuchungen nach Möglichkeit entnommen werden - sind vollständige Abfolgen also eher zu erwarten. Daß andererseits bei insgesamt flachen Hohlformen selbst im Tiefsten mit einem Hiatus gerechnet werden muß, zeigen die Befunde aus dem Rabensberg- und Esinger Moor.

Bei Einbruchshohlformen über Toteis, Salz u. dgl. kann aus der heutigen Tiefenlage der Sedimente nur sehr bedingt auf die zur Zeit ihrer Bildung geschlossen werden. Was heute tief liegt, kann in ganz flachem Wasser abgesetzt worden sein. [So fand etwa AvERDIEcK (1978) im Plöner See bei $41 \mathrm{~m}$ Wassertiefe als ältestes limnisches Sediment offenbar ufernah gebildete Absätze aus Dryas-3.] Profilentnahme in tieferen Teilen eines Sedimentationsraumes ist also nur dann Garantie für \pm kontinuierlich gewachsene Sedimente, wenn Tief wasserbedingungen für deren Bildungszeit nachgewiesen werden können.

Wenn die Beobachtungen im Kubitzbergmoor richtig gedeutet worden sind und sich verallgemeinern lassen, kann für die Umlagerungsvorgänge während bzw. nach der Hiatus-Bildung das in Abb. 5 wiedergegebene Modell angenommen werden. Während also im Tieferen der Seen Absatz von Dryas-3-Sedimenten bereits wieder eingesetzt hatte (oder die Sedimentation auch gar nicht unterbrochen war), erfolgte im Flacheren noch Erosion von Alleröd-Sediment. Dieses gelangte mit seinen zumeist hohen Pollengehalten in Dryas-3-Absätze.

Die durch erhöhte Anteile an organischer Substanz dunklen Lagen im Dryas-3-Silt des Kubitzbergmoores (vgl. Abb. 4) dürften auf diese Weise entstanden sein. - Die im Scharnhagener Moor beobachtete Erscheinung, daß Dryas-3-Silt unmittelbar oberhalb der Alleröd-Algengyttja eine allerödzeitliche Pollen- und Großrestflora führt (vgl. Abschn. 2.2), kann kaum anders gedeutet werden. Der geringe, gegenüber den jüngeren Silten nicht wesentlich erhöhte Glühverlust dieser Lagen (vgl. Abb. 2) ist dann darauf zurïckzuführen, daß bei Umlagerung der Alleröd-Algengyttja im $\mathrm{O}_{2}$-reichen Dryas-3-Wasser die Algenschleime zersetzt wurden, nicht aber Pollen und Großreste. - An anderen Lokalitäten, wo Dryas-3-Sedimente über größere Mächtigkeit bedeutende Anteile an Alleröd-Material enthalten und eine allerödzeitliche Pollenflora führen (vgl. Usınger 1975: 149), scheint dagegen Erosion telmatischer Alleröd-Torfe durch erhöhte Dryas-3Wasserstände Ausgangspunkt der Umlagerung gewesen zu sein. - 
Wie oben erläutert, ist der unvermittelte Übergang zwischen Alleröd- und Dryas-3Sediment das wesentliche und oft einzige lithostratigraphische Merkmal des Hiatus. In manchen Fällen ist dieses Merkmal überaus deutlich, in anderen eher unauffällig. Es muß damit gerechnet werden, daß es durch höhere Anteile umgelagerten Alleröd-Materials im ältesten Dryas-3-Sediment völlig verwischt sein kann.

Nur ein Teil der Pollendiagramme zeigt im Bereich des Hiatus die erwarteten sprunghaften Häufigkeitsveränderungen, andere lassen keine oder nur gleitende Veränderungen erkennen: Umlagerung im Zuge der Bildung des Hiatus hat auch dessen pollenanalytisch faßbare Merkmale zerstört.

So sind Profile denkbar, bei denen selbst ein ausgedehnter Hiatus weder lithostratigraphisch noch pollenanalytisch unmittelbar erkannt werden kann.

\section{Schriftenverzeichnis}

Ammann-Moser, B. (1975): Vegetationskundliche und pollenanalytische Untersuchungen auf dem Heidenweg am Bielersee. - Beitr. geobot. Landesaufn. d. Schweiz, 56: 76 pp.; Bern.

Andersen, S. T. (1980): Early and Late Weichselian chronology and birch assemblages in Denmark. - Boreas, 9: 53-69; Oslo.

Averdieck, F.-R. (1978): Palynologischer Beitrag zur Entwicklungsgeschichte des Großen Plöner Sees und der Vegetation seiner Umgebung. - Arch. Hydrobiol., 83: 1-46; Stuttgart.

- \& Prange, W. (1975): Palynologische und tektonische Untersuchungen einer von Toteis gestörten Schichtfolge am Hochfelder See bei Bothkamp (Holstein). - Meyniana, 27, 1-13; Kiel.

Berglund, B. E. (1966): Late-Quaternary vegetation in eastern Blekinge, southeastern Sweden. A pollen-analytical study. I. Late-Glacial time. - Opera Botan., 12, 1: 180 pp.; Stockholm.

Beug, H.-J. (1976): Die spätglaziale und frühpostglaziale Vegetationsgeschichte im Gebiet des ehemaligen Rosenheimer Sees (Oberbayern). - Bot. Jahrb. Syst., 95: 373-400; Stuttgart.

Brande, A. (1980): Pollenanalytische Untersuchungen im Spätglazial und frühen Postglazial Berlins. - Verh. Bot. Ver. Prov. Brandenburg, 115: 21-72; Berlin.

Cleveringa, P., de Gans, W., Kolstrup, E. \& Paris, F. P. (1977): Vegetational and climatic developments during the Late Glacial and the early Holocene and aeolian sedimentation as recorded in the Uteringsveen (Drente, The Netherlands). - Geol. Mijnbouw, 56, 3: 234242; s'Gravenhage.

Degerbøl, M. \& Krog, H. (1959): The reindeer Rangifer tarandus L.) in Denmark. - Biol. Skr. Dan. Vid. Selsk., 10, 4: 165 pp.; København.

Dietz, C., Grahle, H.-O \& Müller, H. (1958): Ein spätglaziales Kalkmudde-Vorkommen im Seck-Bruch bei Hannover. - Geol. Jb., 76: 67-102; Hannover.

Firbas, F. (1954): Die Vegetationsentwicklung im Spätglazial von Wallensen im Hils. - Nachr. d. Akad. d. Wissensch. in Göttingen; Math.-physik. Klasse, IIb Biol.-physiol.-chem. Abt.: 37-50; Göttingen.

Frenzel, B. (1967): Die Klimaschwankungen des Eiszeitalters. — Die Wissenschaft, 129: 291 pp.; Braunschweig.

Grube, F. (1957): Das Oberflächenbild der Salzstöcke Elmshorn, Lägerdorf (Holstein) und Stade (Niedersachsen). - Mitt. Geol. Staatsinst. Hamburg, 26: 5-22; Hamburg.

Hallik, R. \& Grube, E. (1954): Spät- und postglaziale Gyttja im Altmoränengebiet bei Elmshorn. - Neues Jb. Geol. Paläontol., Mh., 7: 315-322; Stuttgart.

van der Hammen, T. (1953): Late-Glacial flora and periglacial phenomena in The Netherlands. — Leidse Geol. Meded., 17: 71-184; Leiden.

Iversen, J. (1942): En pollenanalytisk Tidfaestelse af Ferskvandslagene ved Nørre Lyngby. Med Bemaerkninger om de senglaciale Naturforhold i Danmark. - Medd. Dansk. Geol. Foren., 10, 2: 130-151; København.

- (1954): The Late-Glacial flora of Denmark and its relation to climate and soil. - Danm. Geol. Unders. II. raekke, 80: 87-119; København. 
Krog, H. (1954): Pollen analytical investigation of a C14-dated Alleröd-section from RudsVedby. - Danm. Geol. Unders. II. raekke, 80: 120-139; København.

Lundovist, G. (1924): Sedimentationstyper i insjöarna. - Geol. Fören. Stockh. Förh., 46, 1/2: 56-75; Stockholm.

Müller, H. (1953): Zur spät- und nacheiszeitlichen Vegetationsgeschichte des mitteldeutschen Trockengebietes. - Nova Acta Leopold. N. F., 16, 110: 67 pp.; Leipzig.

- (1969): Diskordanzen und Umlagerungserscheinungen in holozänen Sedimenten flacher Seen Nordwestdeutschlands. - Mitt. Internat. Verein. Limnol., 17: 211-218; Stuttgart.

Müller, H. M. (1961): Ein Pollendiagramm aus dem Äppelbruch bei Eberswalde. - Arch. f. Forstwesen, 10: 809-816; Berlin.

- (1961): Pollenanalytische Untersuchungen im Bereich des Meßtischblattes Thurow/Südostmecklenburg. - Diss. Halle/S. (Math.-naturw. Fak.). 203 S.; Halle/S.

- (1970): Die spätglaziale Vegetationsentwicklung in der DDR. - In: Quartärkomitee der DDR (Hrsg.): Probleme der weichsel-spätglazialen Vegetationsentwicklung in Mittel- und Nordeuropa: 81-109; Frankfurt/Oder (Quartärkomitee der DDR).

Nichols, H. (1967): The suitability of certain categories of lake sediments for pollen analysis. Pollen et Spores, 9: 615-620; Paris.

Overbeck, F. (1975): Botanisch-geologische Moorkunde. - 719 pp.; Neumünster (Wachholtz).

SchütrumpF, R. (1936): Paläobotanisch-pollenanalytische Untersuchungen der paläolithischen Rentierjägerfundstätte von Meiendorf bei Hamburg. - Veröff. Archäol. Reichsinst., 1: 1-54; Neumünster.

StEinberG, K. (1944): Zur spät- und nacheiszeitlichen Vegetationsgeschichte des Untereichsfeldes. - Hercynia, 3: 529-587; Halle/S.

Tidelski, F. (1960): Pollenanalytische Untersuchungen von voll-, spät- und postglazialen Ablagerungen aus dem Trentmoor und dem Brennacker (Krs. Plön). - Schr. Naturw. Ver. Schlesw.Holst., 30: 92-109; Kiel.

Troels-Smith, J. (1955): Karakterisering af løse jordarter. - Danm. Geol. Unders. IV. raekke, 3, 10: 73 pp.; København.

Usinger, H. (1975): Pollenanalytische und stratigraphische Untersuchungen an zwei SpätglazialVorkommen in Schleswig-Holstein. - Mitt. Arb.-Gem. Geobot. Schlesw.-Holst. Hambg., 25: 183 pp.; Kiel.

- (1978a): Bölling-Interstadial und Laacher Bimstuff in einem neuen Spätglazial-Profil aus dem Vallensgård Mose/Bornholm. Mit pollengrößenstatistischer Trennung der Birken. - Danm. Geol. Unders. Årbog, 1977: 5-29; København.

- (1978b): Pollen- und großrestanalytische Untersuchungen zur Frage des Bölling-Interstadials und der spätglazialen Baumbirken-Einwanderung in Schleswig-Holstein. - Schr. Naturw. Ver. Schlesw.-Holst., 48: 41-61; Kiel.

- (1981): Zur spät- und frühen postglazialen Vegetationsgeschichte der schleswig-holsteinischen Geest nach einem Pollen- und Pollendichtediagramm aus dem Esinger Moor. - Pollen et Spores; Paris. - [Im Druck].

Wasylikowa, K. (1964): Vegetation and climate of the Late-Glacial in Central Poland based on investigations made at Witów near Łeczyca. - Biul. Perygl., 13: 261-421; Łódź.

Wijmstra, T. A. (1978): Palaeobotany and climatic change. - In: J. Gribbin (ed.): Climatic change: 25-45; Cambridge (Cambridge University Press). 
